T-43

\section{A new regulator of mitochondrial OXPHOS in hepatocytes and HCC cell lines: the AAA+ ATPase RuvBL1}

I. Simeone ${ }^{1,2}$, M. Materozzi ${ }^{1,2}$, E. Ceni ${ }^{1}$, S. Polvani ${ }^{1}$, M. Tarocchi $^{1}$, A. Galli ${ }^{1}$, T. Mello $^{1}$

${ }^{1}$ Department of Experimental and Clinical Biomedical Sciences "Mario Serio", University of Florence, Florence, Italy

${ }^{2}$ GenOMeC Doctorate, University of Siena, Siena, Italy

Hepatocellular carcinoma (HCC) is currently a major challenge in medicine for its poor prognosis and lack of effective therapeutic options. The AAA+ ATPase RuvBL1 associates with Hsp90 in several multiprotein complexes regulating key cellular pathways such as cell proliferation, gene expression, chromatin remodeling and telomere maintenance. In several human cancer, including HCC, RuvBL1 overexpression correlates with a poor prognosis. Despite the several functions potentially regulated by RuvBL1, its role in the onset and progression of HCC is still unknown. We had previously generated a RuvBL1 hepatocyte-specific knock-out mouse model and observed by TEM major structural alterations in mitochondria of hepatocytes. Moreover, by a 2D-proteomic screening, we found that several key mitochondrial proteins and Hsp90 $\beta$ were significantly reduced in RuvBL1-silenced hepatoma cells in vitro.

Therefore, we aimed to dissect the role of RuvBL1 as a potential regulator of mitochondria functionality in HCC cells.

First, we found that RuvBL1 targeting by RNAi significantly impairs mitochondrial respiration (OXPHOS) in normal hepatocytes (AML-12) and hepatoma cell lines (Hepa1-6, HepG2, Huh7, Hep3B). Live-cell imaging experiments showed a significant increase of mitochondrial ROS production and mitochondrial depolarization in RuvBL1-silenced cells. We next explored the RuvBL1-Hsp90 axis in mitochondrial functionality. We found that RuvBL1 is a positive regulator of HSP90AB1 promoter and mRNA levels, both in hepatoma and normal hepatocyte cell lines. Interestingly, Hsp90 inhibitors (17-AAG, VER-50589) effectively reduced mitochondrial OXPHOS paralleling RuvBL1 silencing. Analyzing the LIHC-TCGA database, we found a significant correlation between RUVBL1 and HSP90AB1 expression in HCC patients, consistently with our in vitro data. Finally, Gene Ontology analysis performed on genes differentially expressed in high- vs low-RUVBL1 HCC samples in the TGCA database, highlighted a significant enrichment of mitochondria-related terms.

In conclusion, our data uncover a novel role of RuvBL1, possibly through its interaction with Hsp90 $\beta$, in mitochondria integrity and function.

https://doi.org/10.1016/j.dld.2018.11.109
T-44

Surface enhanced Raman spectroscopy as a diagnostic tool for circulating miRNA detection in HCC: the SERMI4cancer project

D. Pascut ${ }^{1}$, A. Bonifacio ${ }^{2}$, A. Visintin ${ }^{3,4}$, A. Di Silvestre ${ }^{2}$, E. Mitri ${ }^{2}$, L.S. Crocè ${ }^{1,3,4}$, C. Tiribelli ${ }^{1}$

${ }^{1}$ Fondazione Italiana Fegato - ONLUS, Centre for Liver Studies, Area Science Park Basovizza, Trieste, Italy

2 Department of Architecture and Engineering, University of Trieste, Trieste, Italy

${ }^{3}$ Departmento of Medical Sciences, University of

Trieste, Trieste, Italy

${ }^{4}$ Clinica Patologie Fegato, Azienda Sanitaria Universitaria Integrata di Trieste (ASUITS), Trieste, Italy

Introduction: Hepatocellular carcinoma (HCC) is one of the most common cancers worldwide with more than 800,000 newly diagnosed cases every year. The late diagnosis, that limits the treatments options, makes this tumor the fifth cause of cancer-related death. The existing diagnostic procedures are expensive and lack of adequate sensitivity and the specificity. The growing interest in the use of circulating miRNAs as reliable biomarkers for cancer diagnosis opens new perspectives in clinical oncology.

Aim: The main objective of the SERMI4Cancer project is to develop a portable, user friendly, and cost-effective Point Of Care (POC) system integrated with the innovative Surface Enhanced Raman Spectroscopy (SERS) technology for circulating miRNA detection in HCC patients.

Materials and methods results: The system allows a label-free direct detection of the analyte making this technology suitable for a POC system for multiple applications. The SERMI4Cancer project includes the development of three components: The miRNA separation and binding support (polyfunctional matrix); the detection system consisting of a Portable Raman Spectrometer and nanostructured SERS Sensor; the data integration system. Molecular beacons targeting miRNAs are immobilized onto silver coated silicon nanopillars spotted on a nitrocellulose membrane and incubated with patients' plasma. Quantifications are performed with the Raman Renishaw spectrometer, a simple, rapid and powerful tool yielding a unique "chemical fingerprint" of the chemical composition of the sample. SERS technology allow a cost-effective detection of very low concentrations of miRNAs (attomolar) increasing the accuracy of the diagnostic biomarker candidate at early stages of the disease.

Conclusion: These results will open new perspectives on HCC diagnosis that could be useful for the development of a point of care system with the innovative SERS technology for miRNAs detection blood samples.

Funding: Supported by the project surface-enhanced Raman microRNA for cancer(SERMI4CANCER) PORFESR 2014-2020 FVG, decree no.3028 05/05/2017 and no.4526 16/06/2017.

https://doi.org/10.1016/j.dld.2018.11.110 